\title{
Measurement of trapped and quasitrapped deuteron populations in PAMELA experiment
}

\author{
S. A. Koldobskiy ${ }^{* 12}$, O. Adriani ${ }^{1,2}$, G. C. Barbarino ${ }^{3,4}$, G. A. Bazilevskaya ${ }^{5}$, R. \\ Bellotti $^{6,7}$, M. Boezio ${ }^{8}$, E. A. Bogomolov ${ }^{9}$, M. Bongi ${ }^{1,2}$, V. Bonvicini ${ }^{8}$, S. Bottai ${ }^{2}$, A. \\ Bruno $^{6,7}$, F. Cafagna ${ }^{7}$, D. Campana ${ }^{4}$, P. Carlson ${ }^{13}$, M. Casolino ${ }^{11}$, G. Castellini ${ }^{14}$, \\ C. De Donato ${ }^{10}$, C. De Santis ${ }^{11}$, N. De Simone ${ }^{10}$, V. Di Felice ${ }^{10}$, V. Formato ${ }^{8, \dagger}$, A. M. \\ Galper $^{12}$, A. V. Karelin ${ }^{12}$, S. V. Koldashov ${ }^{12}$, S. Y. Krutkov ${ }^{9}$, A. N. Kvashnin ${ }^{5}$, \\ A.A. Leonov ${ }^{12}$, V. Malakhov ${ }^{12}$, L. Marcelli ${ }^{11}$, M. Martucci ${ }^{11,15}$, A. G. Mayorov ${ }^{12}$, W. \\ Menn $^{16}$, M Mergè $^{10,11}$, V. V. Mikhailov ${ }^{12}$, E. Mocchiutti ${ }^{8}$, A. Monaco $^{6,7}$, N. Mori $^{2}$, \\ R. Munini ${ }^{8,17}$, G. Osteria ${ }^{4}$, F. Palma ${ }^{10,11}$, B. Panico ${ }^{4}$, P. Papini ${ }^{2}$, M. Pearce ${ }^{13}$, P. \\ Picozza $^{10,11}$, M. Ricci ${ }^{15}$, S. B. Ricciarini ${ }^{14}$, R. Sarkar ${ }^{18, \dagger \dagger}$, V. Scotti ${ }^{3,4}$, M. Simon ${ }^{16}$, R. \\ Sparvoli $^{10,11}$, P. Spillantini ${ }^{1,2}$, Y. I. Stozhkov ${ }^{5}$, A. Vacchi ${ }^{8,19}$, E. Vannuccini ${ }^{2}$, \\ G.I. Vasilyev ${ }^{9}$, S. A. Voronov ${ }^{12}$, Y. T. Yurkin ${ }^{12}$, G. Zampa ${ }^{8}$, N. Zampa ${ }^{8}$ \\ ${ }^{1}$ University of Florence, Department of Physics, I-50019 Sesto Fiorentino, Florence, Italy \\ ${ }^{2}$ INFN, Sezione di Florence, I-50019 Sesto Fiorentino, Florence, Italy \\ ${ }^{3}$ University of Naples "Federico II", Department of Physics, I-80126 Naples, Italy \\ ${ }^{4}$ INFN, Sezione di Naples, I-80126 Naples, Italy \\ ${ }^{5}$ Lebedev Physical Institute, RU-119991, Moscow, Russia \\ ${ }^{6}$ University of Bari, Department of Physics, I-70126 Bari, Italy \\ ${ }^{7}$ INFN, Sezione di Bari, I-70126 Bari, Italy \\ ${ }^{8}$ INFN, Sezione di Trieste, I-34149 Trieste, Italy \\ ${ }^{9}$ Ioffe Physical Technical Institute, RU-194021 St. Petersburg, Russia \\ ${ }^{10}$ INFN, Sezione di Rome "Tor Vergata", I-00133 Rome, Italy \\ ${ }^{11}$ University of Rome "Tor Vergata", Department of Physics, I-00133 Rome, Italy \\ ${ }^{12}$ National Research Nuclear University MEPhI, RU-115409 Moscow \\ ${ }^{13} \mathrm{KTH}$, Department of Physics, and the Oskar Klein Centre for Cosmoparticle Physics, \\ AlbaNova University Centre, SE-10691 Stockholm, Sweden \\ ${ }^{14}$ IFAC, I-50019 Sesto Fiorentino, Florence, Italy \\ ${ }^{15}$ INFN, Laboratori Nazionali di Frascati, Via Enrico Fermi 40, I-00044 Frascati, Italy \\ ${ }^{16}$ Universität Siegen, Department of Physics, D-57068 Siegen, Germany \\ ${ }^{17}$ University of Trieste, Department of Physics, I-34147 Trieste, Italy \\ ${ }^{18}$ Indian Centre for Space Physics, 43 Chalantika, Garia Station Road, Kolkata 700084, West Bengal, India \\ ${ }^{19}$ University of Udine, Department of Mathematics and Informatics, I-33100 Udine, Italy \\ ${ }^{\dagger}$ Now at INFN, Sezione di Perugia, I-06123 Perugia, Italy \\ ${ }^{\dagger}$ Previously at INFN, Sezione di Trieste, I-34149 Trieste, Italy \\ E-mail: sakoldobskiy@mephi.ru
}


The results of measurements of trapped and albedo cosmic ray deuteron fluxes obtained in the PAMELA experiment are presented in this work. The PAMELA is an international experiment aimed for measurements of cosmic ray particle fluxes in wide energy range. In particular, analysis of PAMELA data gives possibility to identify deuterons and to reconstruct deuteron spectra of different origin (galactic cosmic ray, re-entrant albedo and radiation belt particles). The first results of trajcectory reconstruction for trapped and albedo deuterons are presented in this work. This investigation was done by solving equations of particle motion in Earth's magnetic field by means of numerical integration methods.

The 34th International Cosmic Ray Conference, 30 July- 6 August, 2015

The Hague, The Netherlands

* Speaker. 


\section{Introduction}

The particles registered in near Earth orbit belong to three different populations. There are galactic cosmic rays (GCR) particles generated in cosmic ray sources or different interaction processes in Galaxy, albedo particles originated from interactions of high energy cosmic rays with the nuclei of the residual atmosphere layer and radiation belt particles trapped by magnetic field. Depending on the particle registration point (in terms of geomagnetic coordinates) and on its energy it is possible to consider that particle belongs to one or another of aforementioned populations.

The deuteron fluxes of different origin were measured in some experiments in past.

The galactic deuterons were registered for the first time in 1960's by the IMP-III experiment [1]. The most accurate results were obtained later in series of BESS experiments (see for example, [2]), AMS-01 experiment [3] and in the PAMELA experiment [4].

For the first time the registration of deuteron albedo radiation was mentioned in [5, 6]. The results of the unique attempt to measure the spectrum of deuterons under radiation belt in the AMS-01 experiment were presented in [3]. The last and the most accurate measurements were implemented by the PAMELA experiment [7].

At last low energy trapped deuterons were detected in SAMPEX [6] and NINA experiments [8].

In this paper we present the first results of deuteron trajectory reconstruction procedure and identification of different populations of deuterons obtained by analysis of PAMELA experimental data for period from July 2006 till January 2008.

\section{PAMELA experiment}

PAMELA instrument [9] (Fig. 1) is a magnetic spectrometer (MS), composed by a permanent magnet hosting a tracking system and equipped with a time-of-flight system (ToF) and the calorimeter. The tracking system consists of six double-sided micro-strip silicon sensors. MS allows to measure the particle charge and rigidity (momentum over charge) with high precision. ToF system consists of three double-layer plastic scintillators $\left(S_{1}, S_{2}\right.$ and $\left.S_{3}\right)$ and serves for precise measurements of particle velocity and energy losses in each detector. The total depth of calorimeter is 16.3 radiation lengths, and the main function of calorimeter is high energy lepton-hadron separation. PAMELA apparatus also includes a tail shower scintillation detector $S_{4}$ and neutron detector. The magnet is surrounded by scintillation counters operating in the anticoincidence mode (AC) used to select events when particles entering in the spectrometer field of view.

PAMELA instrument was installed on board Resurs DK-1 satellite, which was launched 15 June 2006.

Detailed description of the PAMELA detectors can be found in $[9,10,11,12]$.

\section{Particle identification}

The selection of "good" events was carried out at the first stage of data analysis because only the events with correct detector responses could be used for subsequent event identification procedure. The special criterion set for implementation of the so-called "basic" selection has been developed. Events that passed "basic" selection have no signals from anticoincidence detectors. 


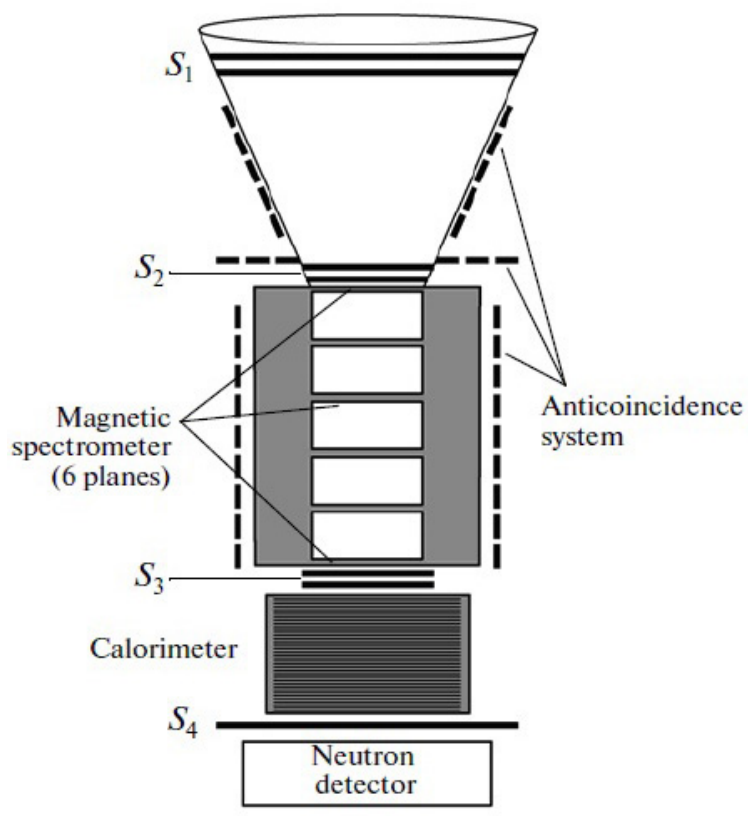

Figure 1: The schematic view of PAMELA experiment.

They corresponded to particle arriving inside the aperture of apparatus, with no interactions in the detector material, and having well approximated trajectory in MS. For the purpose of identification the analysis of the event characteristics was done to separate hydrogen isotopes from helium ones in the energy range from $70 \mathrm{MeV} /$ nucleon to $2000 \mathrm{MeV} /$ nucleon. The most important and complicated task was the authentic identification of deuterons on the huge background of protons with similar detector signals. For the identification of detected particles, as well as the recovery of its energy the multivariate correlation analysis of the signals from different detectors was used. The procedure of event identification was applied using values of particle rigidity $R$, measured by MS, its velocity $\beta$, measured by ToF and multiple measurements of the energy losses of ToF and tracker detectors. The detailed description of the identification method are given in $[4,7,12]$. This identification method was updated because trajectory reconstruction in Earth 's magnetic field requires reliable separation of each deuteron event because if the type of particle will be determined mistaken, the trajectory will be reconstructed wrong as well. So the criteria for selection of deuterons were made stricter in comparison with the selection criteria for spectra reconstruction and only deuterons with kinetic energy from 70 to $400 \mathrm{MeV}$ /nucleon were selected from investigated data sample.

Measured magnetic rigidities were corrected for the energy losses in ToF and MC, evaluated with Monte-Carlo GEANT4 [13] simulations.

\section{Deuteron trajectory reconstruction routine}

Knowing the spacecraft position and orientation with high precision as well as an good angular resolution of instrument allow to measure the particle arriving direction with good accuracy. Using spacecraft orientation data, the particle rigidity and velocity vector provided by magnetic 
spectrometer, the deuteron trajectories were reconstructed in the Earth's magnetosphere by means of a tracing program based on solving the equations of particle motion in Earth's magnetic field by numerical integration methods [14], using the geomagnetic field model International Geomagnetic Reference Field (IGRF) [15]. Maximum value of drift time was chosen to be 10 seconds.

Trajectories were traced back and forth from the registration point until:

1. they reached the boundaries of Earth's magnetosphere during backtracing; these particles were classified as GCR deuterons;

2. they intersected the boundaries of "dense" atmosphere, which was assumed at an altitude of $30 \mathrm{~km}$; these particles were classified as re-entrant albedo deuterons;

3. they survived more than 10 seconds and satisfied to adiabatic conditions; these particles were classified as deuterons of radiation belt.

\section{Trajectory reconstruction results}

Data sample from July 2006 to January 2008 was used and more than 82.000 deuteron events with energies from 100 to $400 \mathrm{MeV} /$ nucleon were selected.

In the Figure 2 the randomly selected trajectories of 400 deuterons are reconstructed in 3D and $2 \mathrm{D}$ pole views. The galactic cosmic ray deuterons entering the spectrometer in the pole regions are clearly visible. Albedo deuterons originated from Earth atmosphere as well as belt of trapped deuterons are seen. One can see an interesting fact that the belt of "quasitrapped" albedo deuterons is visible as well.

In the Figure 3 geographic coordinates of deuteron absorption in Earth's atmosphere for galactic cosmic rays are shown. They can be detected only at pole regions where they move along the Earth magnetic field lines for studied energy range. Generated in this geomagnetic zones albedo particles can not be registered by PAMELA, because the PAMELA main axis directed to zenit, while generated albedo particles moving from Earth along the lines of magnetic field so they can not arrive in the PAMELA axis. Only GCR deuterons with rigidity above Stroemer geomagnetic
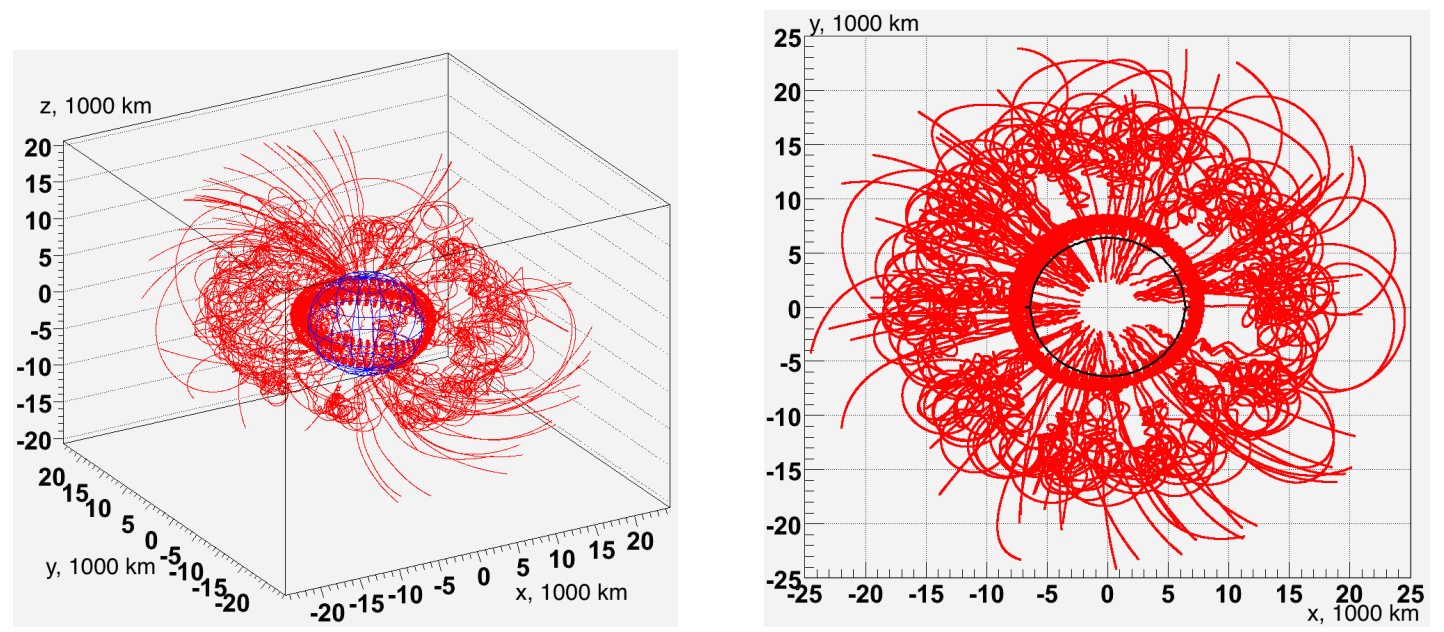

Figure 2: Reconstructed trajectories for deuterons of different populations. 

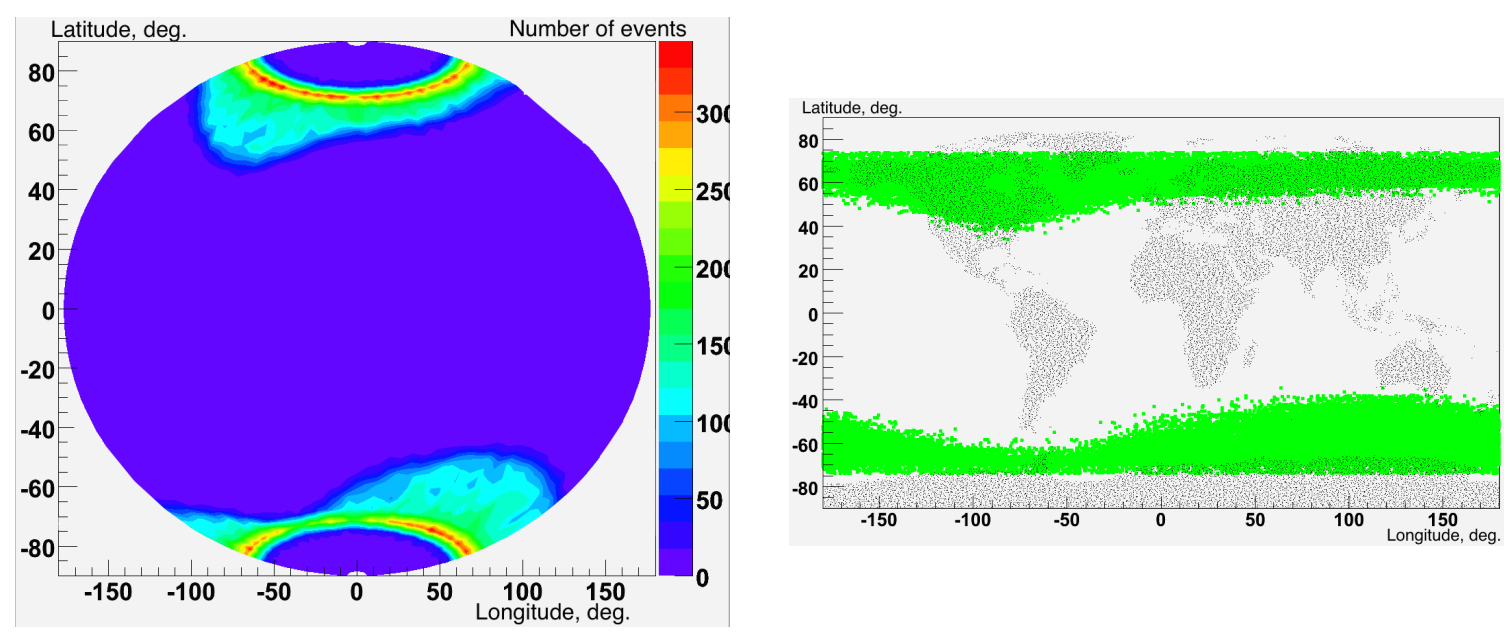

Figure 3: Geographic coordinates of GCR deuterons absorption in Earth's atmosphere.

cutoff $R=14.9 / L^{2}$ [16], where $L$ is an McIlwain shell parameter, can be registered here. The features of GCR deuterons spectrum are discussed in other work [4].

In the Figure 4 the coordinates of RB deuterons after 10 seconds of backtracing are shown. These particles are registered in zone of Brazilian Magnetic Anomaly (BMA) because trajectory of PAMELA flight intersects with radiation belt fluxes in this zone. These particles are moving in Earth's magnetic field for a long time and they form the Earth's radiation belt with other CR species (mostly, protons). There is the interesting fact that observed maximum rigidity of radiation belt deuterons is about $1.8 \mathrm{GV}$, that corresponds to kinetic energy about $360 \mathrm{MeV} /$ nucleon.

Finally, in the Figure 5 points of deuterons generation for re-entrant albedo particles are shown. These particles are registered mostly on all route of spacecraft with exception for polar zones and the zone of BMA. For re-entrant albedo deuterons the distribution for lifetime was constructed (Fig. 6a). It clearly visible from this Figure, that $90 \%$ of re-entrant albedo have lifetime less than 2 seconds and these events are absorbed quickly by Earth's atmosphere. In contrary, small
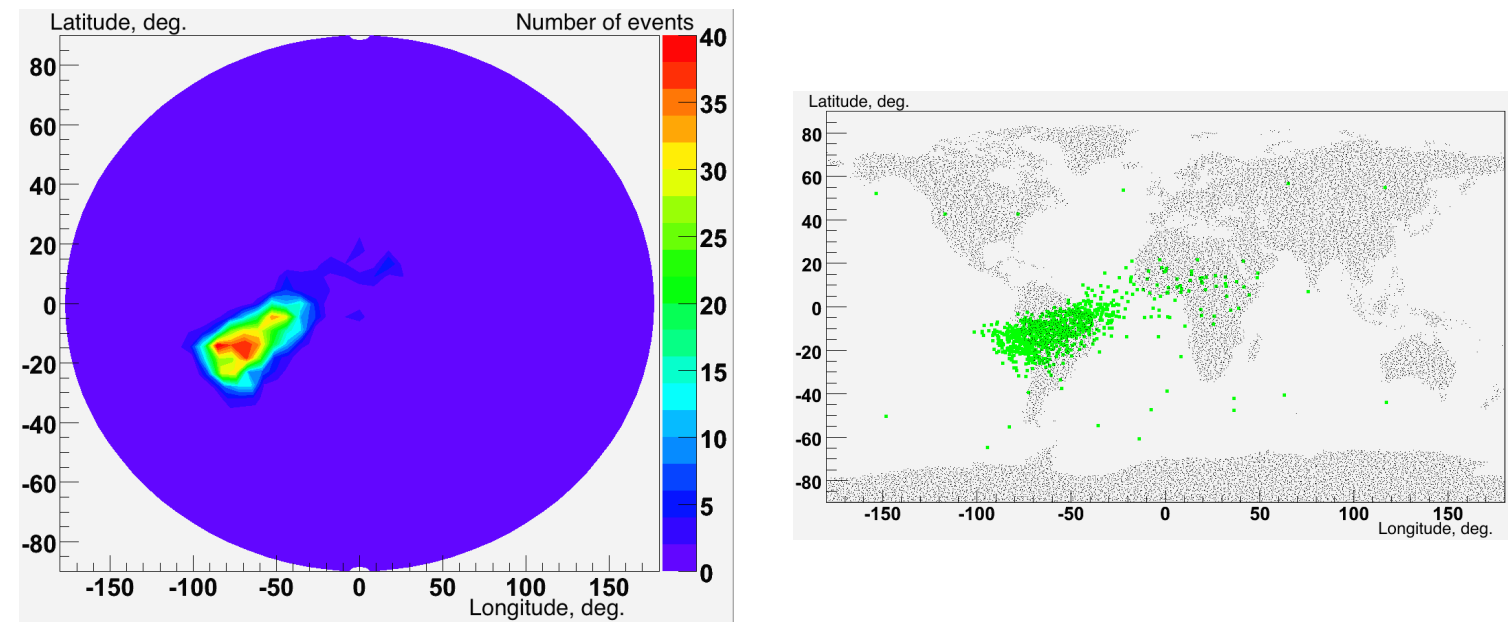

Figure 4: Geographic coordinates of radiation belt deuterons after 10 seconds of backtracing. 

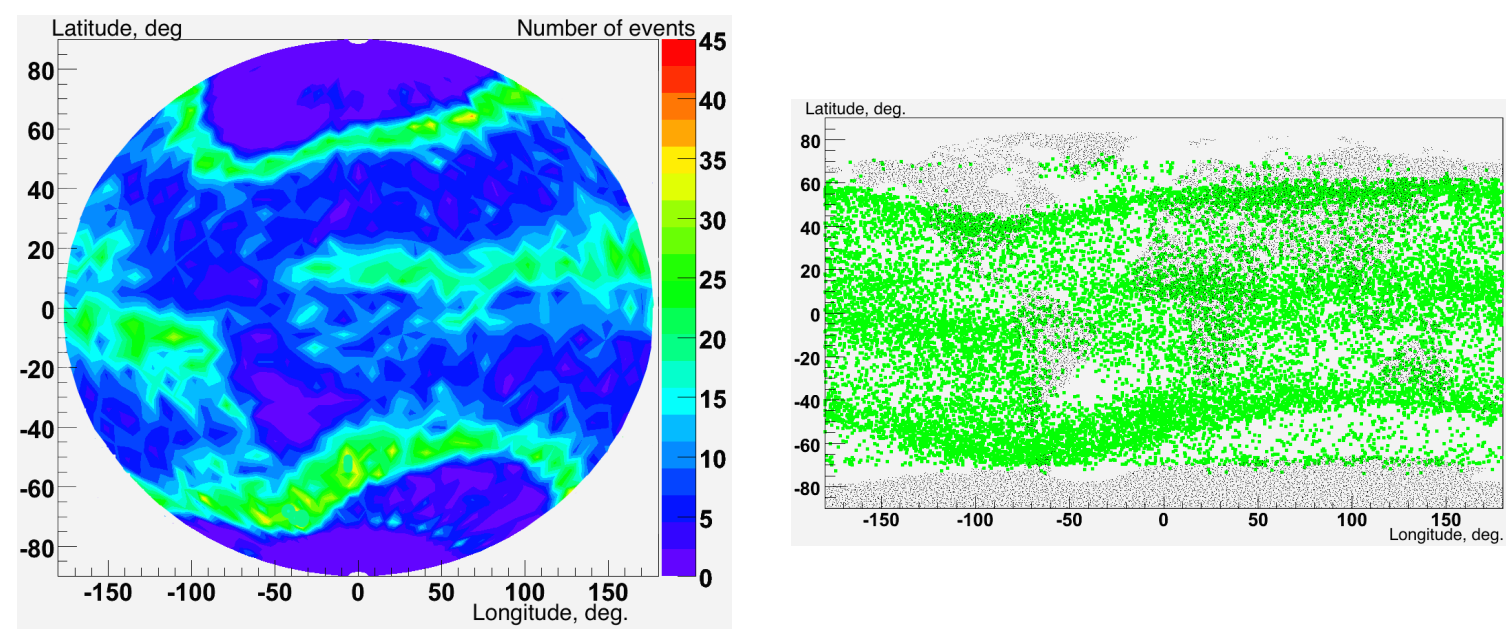

Figure 5: Geographic coordinates of absorption points for re-entrant albedo deuterons in Earth's atmosphere.

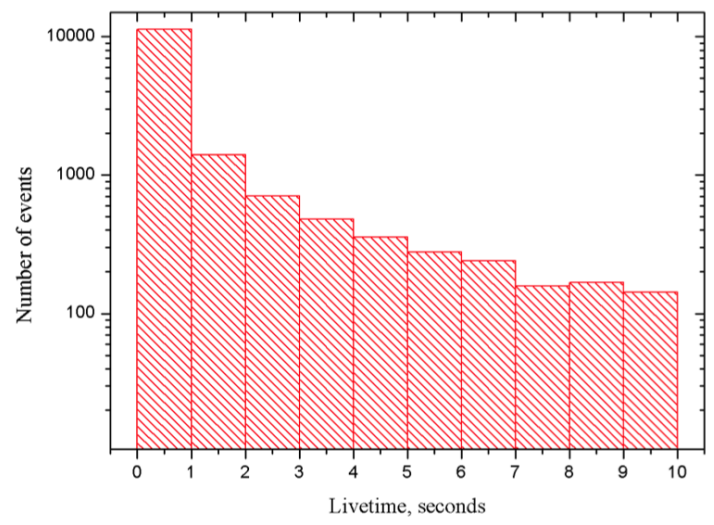

(a)

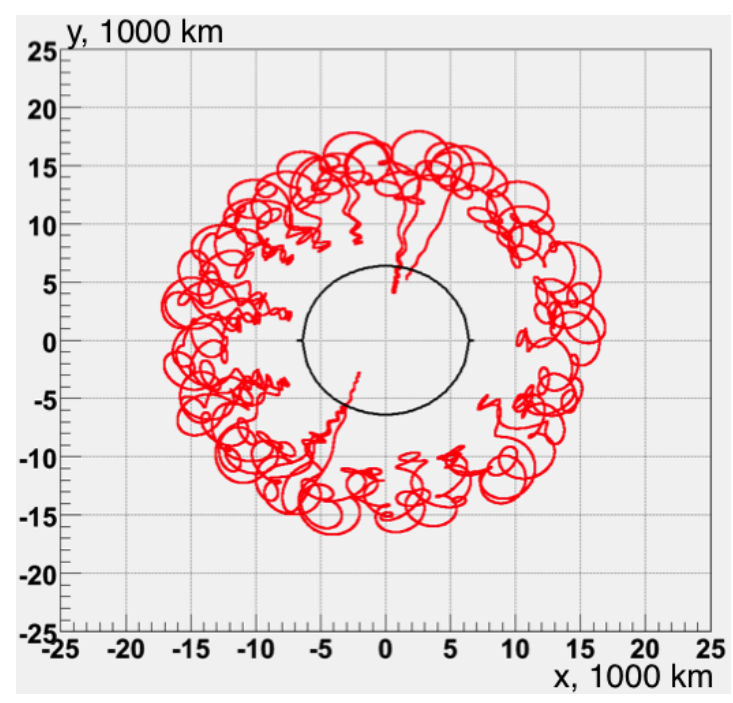

(b)

Figure 6: (a) The distribution of lifetime for observed re-entrant albedo deuterons;

(b) The typical trajectory of "quasitrapped" trajectory for re-entrant albedo deuteron with lifetime of 9.8 seconds.

fraction of events has relatively big lifetimes (up to 10 seconds). These particles have interesting "quasitrapped" trajectories (Fig. 6b) in Earth's magnetic field and they can form a belt of "quasitrapped" deuterons.

\section{Conclusion}

As a result of this work the trajectory reconstruction of GCR, albedo and radiation belt deuterons with kinetic energies from 70 to $400 \mathrm{Mev} /$ nucleon registered in PAMELA experiment was per- 
formed. Three populations of particles with completely different features of motion in Earth 's magnetic field were completely distinguished. Points of generation and absorption for this particles were reconstructed. Interesting fact that a small fraction of "quasitrapped" re-entrant particles is observed. These particles are moving in Earth magnetic field with complicated trajectories. Results of trajectory reconstruction approves that the geomagnetic selection used for spectrum reconstruction in previous works was correct absolutely.

\section{Acknowledgments}

This work was carried out with financial support of the Russian Science Foundation (grant 14-12-00373) and the grant of the President of the Russian Federation MK-4599.2014.2.

\section{References}

[1] Simpson, J.A., Fan, C.Y. and Gloeckler, G., Physical Review Letters, 1966, No. 17, p. 329.

[2] Wang, J. Z., Seo, E. S., Anraku, K. et al. Astrophysical Journal, 2002, V. 564 (1), p. 244.

[3] G. Lamanna et al., in proceedings of the 27th International Cosmic Ray Conference, 2001, p. 1614.

[4] Adriani, O., Barbarino, G.C., Bazilevskaya, G.A. et al., Astrophysical Journal, 2013, V. 770 (1), p. 2.

[5] Looper, M.D., Blake, J.B., Mewaldt, R.A., Advances in Space Research, 1998, V. 21(12), p. 1679..

[6] Looper, M.D., Blake, J.B., Cummings, J.R., Mewaldt, R.A., Radiation Measurements, 1996, V. 26, p. 967.

[7] Koldobskiy, S., Adriani, O., Barbarino, G.C., et al., Bulletin of the Russian Academy of Sciences: Physics, V. 79 (3), pp. 294-297.

[8] Bakaldin, A., Galper, A., Koldashov, S., et al., Journal of Geophysical Research: Space Physics, 2002, V. 107, I. A8, p. SMP 8-1.

[9] Picozza, P., Galper, A.M., Castellini G. et al., Astroparticle Physics, 2007, V. 27, p. 296.

[10] Ricciarini, S. et al., Nuclear Instruments and Methods in Physics Research A, 2008, V. 582, p. 892.

[11] Papini, P., Adriani, O., Ambriola, M. et al., Nuclear Instruments and Methods in Physics Research A, 2008, V. 588, p. 259.

[12] Voronov, S.A., Danil'chenko, I.A., Koldobskiy, S.A., Instruments and Experimental Techniques, 2011, V. 54, p. 752.

[13] Agostinelli, S., et al., Nuclear Instruments and Methods A, 2003, V. 506, p. 250.

[14] Smart, D. F., Shea, M. A., Advances in Space Research, 2005, V. 36, p. 2012.

[15] Finlay, C. C., Maus, S., Beggan C. D. et al., Geophysical Journal International, V. 183, p. 1216.

[16] Hofmann, D.J., Sauer, H.H. Space Science Reviews, 1968, V. 5-6, No. 8, p. 750. 